\title{
Responsabilités en souffrance. Les conflits autour de la souffrance psychique des salariés d'EDF-GDF (1985-2008).
}

\author{
Version finale (février 2010)
}

\author{
Auteurs : \\ Marlène Benquet, Pascal Marichalar, Emmanuel Martin \\ Doctorants au Centre Maurice Halbwachs (UMR 8097) \\ 48 boulevard Jourdan 75014 Paris \\ tél : 06.72.07.56.21 (MB) / 06.50.83.86.44 (PM) / 06.62.47.96.54 (EM) \\ marlenebenquet@hotmail.com \\ pascal.marichalar@gmail.com \\ emmanuel.martin@ens.fr
}

\section{Résumé en français :}

L'article analyse le traitement de la souffrance psychique des salariés au sein des entreprises Electricité de France-Gaz de France entre 1985 et 2008, à partir d'un dépouillement exhaustif des procès-verbaux d'une instance nationale. Il entend montrer que l'émergence et les problématisations du thème de la souffrance psychique sur la période ne peuvent être comprises sans faire attention aux débats préexistants sur la responsabilité des accidents, à l'identité des personnes qui le mobilisent, et aux argumentations qu'il permet.

\section{Resumé en anglais :}

The struggle over mental health and its responsibility in the French energy sector.

The article studies the issue of the employees' mental suffering in the French energy companies EDF-GDF between 1985 and 2008. It is based on the thorough analysis of the minutes of a national committee of health and security at work. The paper wishes to show that the rise of the theme of workers' mental health during the period cannot be understood without paying attention to the already existing debates on accident responsibility, to the people who summon this theme, and to the argumentations made possible by summoning this theme. 
Un rapide tour d'horizon des recherches récentes en sciences sociales consacrées aux problèmes de santé liés au travail permet de mettre en évidence deux axes de lecture principaux. Le premier est celui de l'invisibilité de ces problèmes, ou plutôt des mécanismes d' « invisibilisation », de " déni », voire d' « organisation de l'opacité statistique » (Holmes, 2006 ; Gollac et al, 2006 ; Rosental, 2007 ; Omnès et Pitti, 2009). La deuxième grille de lecture est celle de la responsabilité des accidents du travail et des maladies professionnelles et de leur judiciarisation, qui s'est accélérée en France à partir des années 1990 autour des pathologies de l'amiante (Henry, 2000 ; 2003 ; Desmond, 2006 ; sur le cas américain, voir Rosner et Markowitz, 2009). Par l'analyse de la souffrance psychique au sein des entreprises Electricité de France-Gaz de France (EDF-GDF), nous proposons de nous placer à l'intersection de ces deux lignes de force : la visibilité et la responsabilité des maux du travail.

Comment étudier sociologiquement l'émergence de débats autour de la souffrance au travail, la dépression et les suicides des salariés ? Une première démarche est de conduire une étude statistique sur le nombre et la cause des suicides de salariés, en s'inscrivant dans la tradition durkheimienne ; elle est rendue difficile par la taille relativement réduite des effectifs étudiés. La deuxième approche, qui semble complémentaire, est celle que nous développons dans cet article : voir comment le thème de la souffrance psychique apparaît au sein des arènes de débat des entreprises, en prêtant l'attention à l'identité des personnes qui les porte, aux objectifs qu'ils poursuivent, ainsi qu'aux langages et corpus théoriques convoqués pour en parler.

Au long de ses vingt-trois ans d'existence (1985-2008), le Comité national d'hygiène, de sécurité et des conditions de travail (CNHSCT) d'EDF-GDF a discuté de ces questions, que l'on regroupera ici sous l'expression générique de « souffrance psychique des salariés ». La mobilisation de catégories telles que la souffrance au travail, les suicides professionnels ou les risques psychosociaux ne s'est pas faite dans le cadre de sessions spécialisées sur le sujet, mais au fil d'une discussion déjà établie depuis longtemps au sein du comité sur les risques professionnels et leur impact sur la santé des salariés (le CNHSCT prend la suite du comité de coordination des comités d'hygiène et de sécurité, qui existe depuis 1955). Les argumentations qui touchent à la santé mentale se sont ajoutées et confrontées à des considérations d'autres natures, au sein de débats dont les enjeux dépassaient la simple recherche technique d'une méthode de prévention efficace.

N'étant ni médecins, ni psychologues, il ne nous appartient pas de nous prononcer sur l'existence, les formes ni les causes de la souffrance psychique (Dodier, 1999). Dans la lignée des travaux d'Elliot Freidson, nous nous en tenons ici à une "suspension du jugement» sur les dimensions psychologiques et médicales de l'objet "souffrance psychique », pour n'explorer que les aspects sociaux de la mobilisation de cette catégorie (Freidson, 1984).

L'étude des quelque six mille pages de procès-verbaux de réunions du CNHSCT montre que la problématique de la souffrance psychique s'est insérée dans un débat préexistant, qui portait sur la part respective de responsabilité de l'employeur et du salarié dans les accidents du travail. Au long de la période étudiée, les enjeux de ce débat ont perduré; les arguments mobilisés par les représentants des salariés d'une part, les représentants des directions de l'autre, se sont en revanche transformés. La problématique de la souffrance psychique s'est insérée parmi ces arguments, avec un succès qui a varié selon deux facteurs : l'identité de ceux qui ont porté cette problématique et les ressources qu'ils pouvaient mobiliser pour la défendre; ainsi que la portée des argumentations que les concepts, langages, corpus théoriques mobilisés, rendaient possibles.

L'histoire du traitement de la souffrance psychique chez EDF-GDF est celle de la reprise en main par la direction des problématiques de santé des salariés, au détriment de l'instance représentative du personnel. Si la souffrance psychique est d'abord intégrée en tant que facteur d'accidents du travail, dans un débat préexistant portant sur la responsabilité des 
accidents graves, les suicides la constituent elle-même en risque lourd, qui questionne directement la responsabilité de l'employeur. L'usage par la direction du concept de risques psychosociaux est une forme de reconnaissance du problème; elle contribue cependant à court-circuiter le CNHSCT, remplacé par des structures à la vocation résolument technique plutôt que politique, qui donnent bien moins de poids aux représentants syndicaux et à la recherche de responsabilité.

\section{5-2008: le Comité national d'hygiène, de sécurité et des conditions de travail d'EDF-GDF}

Dès la nationalisation du 8 avril 1946, les établissements EDF et GDF sont deux entités distinctes sur le plan juridique, mais qui comportent néanmoins de nombreux services communs, dont notamment les services de prévention et de médecine. En 1955 naît la commission de coordination des comités d'hygiène et de sécurité (CCCHS), qui chapeaute pendant trente ans les comités hygiène et sécurité d'EDF et de GDF. La loi Auroux du 23 décembre 1982 supprime ces derniers, y substituant les comités d'hygiène, de sécurité et des conditions de travail (CHSCT). L'une des conséquences de l'application de cette loi chez EDF-GDF est la création d'un CHSCT national, en lieu et place de l'ancienne commission de coordination (juillet 1985).

Sont membres de droit du CNHSCT un président, nommé par la direction, et douze représentants du personnel, répartis entre les organisations syndicales selon leur représentativité respective en fonction des résultats des élections professionnelles. Il faut y ajouter des membres à titre consultatif, sans droit de vote : représentants des différentes directions opérationnelles (ou directions " des métiers ", c'est-à-dire des grandes branches des deux entreprises : production, transport, distribution...), représentants des médecins du travail, chefs des services prévention et sécurité. Les représentants du personnel élisent un secrétaire, qui est l'interlocuteur principal du président. L'instance n'a connu que deux secrétaires, tous deux membres de la Confédération générale du travail (CGT) qui a toujours été très largement majoritaire (le plus souvent, elle détenait 9 des 12 sièges syndicaux) : Michel de Coninck de 1985 à 1998, et Joseph Boyer de 1998 à 2008.

Le CNHSCT est conçu sur le modèle des CHSCT. Comme ces derniers, sa création répond au double objectif de prolongation dans l'entreprise de l'action de l'inspection du travail et de développement d'une culture de la prévention des risques professionnels (Hatzfeld, 2006a). L'instance a pour mission commune avec les CHSCT la conduite d'enquêtes consécutives aux accidents du travail et maladies professionnelles graves, l'inspection des sites en vue du repérage des risques, le contrôle de l'instruction des services de sécurité et le développement du sens du risque professionnel. Elle a comme particularité d'être une personne morale - donc de pouvoir attaquer en justice - ainsi que de pouvoir demander des expertises. Chez EDF-GDF, « le CNHSCT détient une compétence propre et une compétence de coordination pour l'ensemble des attributions de la filière CHSCT, mais celles-ci ne sont mises en œuvre que dans la mesure où la décision à prendre relève des Directions Générales ou des Directions opérationnelles »" Théoriquement et juridiquement pensée comme une "instance aux compétences strictement délimitées aux enjeux techniques du suivi des risques et de leur prévention » (Hatzfeld, 2006b p : 413), elle est traversée des mêmes tensions que les CHSCT entre technicité et représentativité (Hatzfeld, 2006b p : 409). La mission technique du CNHSCT est prise en charge par des représentants du personnel et de la direction aux intérêts structurellement distincts, et se trouve donc encadrée de fait par deux autorités et deux types de légitimités. Fréquemment, les débats internes au CNHSCT reconduisent donc sur le terrain de la santé et de la prévention des risques les positions préalablement constituées des différents intervenants.

Les témoignages des anciens membres attestent du caractère solennel du CNHSCT, qui découlait aussi bien de la présence de nombreux directeurs, des dimensions imposantes de la salle (dans laquelle le président, un temps, était installé sur une estrade), que de la présence de micros et d'une sténotypiste, imposant de surveiller ses propos, puisqu'ils seraient ensuite rendus publics. Les procès-verbaux de CNHSCT étaient imprimés à 8000 exemplaires et diffusés à l'ensemble des membres de CHSCT d'EDF-GDF, et sont donc facilement accessibles. La qualité professionnelle de la prise de notes en sténotypie ainsi que le caractère minime des modifications avant validation permettent de considérer ces archives comme un reflet relativement fidèle des échanges verbaux (transposés à la troisième personne).

\section{5-1998 : Controverses autour des enquêtes psychopathologiques}

La première séance du CNHSCT a lieu en juillet 1985. Lors de ses premières années de fonctionnement, le Comité s'intéresse extrêmement peu à la question de la souffrance

\footnotetext{
${ }^{1}$ Circulaire PERS. 844 du 31 mai 1985.
} 
psychique, prolongeant de fait le désintérêt du CCCHS pour cette question. Les débats portent plutôt sur les explosions de gaz et les électrocutions, que les membres du Comité qualifient volontiers de risques lourds ou graves, par opposition à d'autres maux considérés comme mineurs. Cependant, c'est à la faveur d'une discussion sur les risques graves que s'initie un questionnement syndical sur la « souffrance au travail» des salariés d'EDF-GDF.

\section{L'expertise psychopathologique dans la Distribution (1988-1993)}

Lors de la séance de décembre 1988, l'heure est à l'examen des « accidents graves et significatifs » de 1987, une activité habituelle pour le Comité. Rejetant toute responsabilité dans des accidents du travail survenus dans le secteur de la Distribution ${ }^{2}$, les directions soulignent que les salariés victimes ne portaient pas leurs équipements de protection individuelle, et ne peuvent donc que s'en prendre à eux-mêmes. En réponse, un membre de la délégation CGT évoque une étude récente réalisée par des médecins du travail du groupe, qui propose une explication alternative, faisant appel à des facteurs bien plus globaux :

«1) la multiplicité des réformes de structures éloigne les agents des lieux d'intervention 2) l'insuffisance des effectifs détériore les conditions de travail et peut être génératrice d'accidents ».

Les directions réfutent qu'il y ait lien entre des considérations aussi générales et des accidents du travail particuliers. Cependant, en fin de séance, les organisations syndicales décident à l'unanimité d'adopter un "vœu » ${ }^{3}$ qui demande " une expertise portant sur les incidences que ces facteurs peuvent avoir sur la sécurité, les conditions de travail et la santé des agents ».

En septembre 1989, presque un an plus tard, le médecin-chef du Service général de médecine du travail (SGMT) explique avoir consulté deux spécialistes, l'un en épidémiologie, l'autre en ergonomie : «Les réponses de ces deux spécialistes convergent pour indiquer qu'un problème aussi vaste ne peut être traité par les méthodes ergonomiques ou épidémiologiques ». Ce vide méthodologique - comme si pour ces médecins, la question posée était trop neuve pour ces méthodes éprouvées - amène le président à proposer le report de l'examen de ce dossier à une date ultérieure.

En réponse, la CGT adopte une triple stratégie lors de cette séance : elle insiste sur la gravité des «maladies psychiques qui deviennent les principales maladies de l'activité de service » et peuvent conduire au suicide ; elle montre que la souffrance psychique débouche sur davantage d'accidents du travail « graves »; enfin, elle menace le président d'une action en justice pour délit d'entrave s'il n'accepte pas l'expertise ${ }^{4}$. Le secrétaire CGT du Comité, M. De Coninck cite pour la première fois le nom du professeur C. Dejours ; celui-ci lui aurait déjà fourni un programme d'étude sur cinq centres de la distribution ainsi que des propositions chiffrées.

Il apparaît lors des séances suivantes que les directions sont réticentes à poursuivre dans ce domaine, comme elles l'expliquent en mai 1990, considérant

« que la possibilité d'expertise est liée au constat d'un risque grave révélé ou non par une maladie professionnelle ou un accident du travail. Or, il n'apparaît pas aux Directeurs Généraux que ce soit le cas dans la présente affaire ».

En février 1991, les directions cèdent finalement. Le CNHSCT invite C. Dejours pour présenter sa démarche. Il est acté qu'avant une possible extension, l'étude aura d'abord lieu dans deux centres où les médecins sont prêts à « coopérer » : Sambre-Avesnois et Villejuif.

\footnotetext{
${ }^{2}$ En 1990, la distribution regroupe 92000 des 122000 agents EDF-GDF. Ce sont tous les agents qui s'occupent du réseau électrique depuis les postes haute tension, et du réseau gaz depuis les postes de détente, jusqu'aux compteurs des clients (particuliers ou entreprises).

${ }^{3}$ Le vœu, adopté par la majorité des membres élus et adressé aux directions, n'a pas de force coercitive.

${ }^{4}$ Dès lors que l'employeur s'oppose à la constitution ou au fonctionnement d'une institution représentative du personnel, il est passible d'une sanction pénale (Article L4741-1 du Code du travail).
} 
En mars et octobre 1993, C. Dejours est à nouveau l'invité du Comité : il y expose cette foisci les principaux résultats de ses expertises, qui donnent lieu en parallèle à des restitutions au personnel des sites étudiés. Par la suite, de nombreuses autres collaborations ont lieu entre l'équipe de C. Dejours et les syndicats de salariés d'EDF-GDF, décidées au niveau du Comité national mais aussi, fréquemment, des Comités locaux ${ }^{5}$.

\section{Une théorie qui vient en soutien des positions syndicales}

Alors qu'il n'a que rarement été évoqué jusqu'alors, le thème de la souffrance psychique n'apparaît pas pour autant ex machina. Il est amené dans l'enceinte du Comité par des acteurs particuliers, dans le cadre de séances qui peuvent être considérées comme les étapes successives d'une longue discussion entre représentants du personnel et des directions. Le destin de ce thème dans les débats du CNHSCT se comprend à la lumière de deux facteurs : le circuit de diffusion de la théorie psychopathologique du travail, ainsi que les ressources argumentatives nouvelles offertes aux membres du Comité par ce corpus théorique.

C'est incontestablement par les médecins du travail du groupe que la psychopathologie du travail arrive à EDF-GDF. Pendant les années 1980, de nombreux médecins du travail ont été formés à la psychopathologie du travail dans le cadre du Diplôme d'études approfondies du Conservatoire national des arts et métiers, où enseigne C.Dejours (Salman, 2008). Ce pic de formation suit de peu la création des CHSCT, dont l'une des innovations majeures, par rapport aux CHS, est la possibilité offerte aux élus de demander une expertise. C'est donc tout naturellement que de nombreux médecins du travail d'EDF-GDF se mettent à conseiller que les éventuelles expertises soient confiées à l'équipe de C.Dejours ${ }^{6}$. Dans le cadre du Comité national, on note également un transfert de connaissances entre certains de ces médecins et les membres élus, facilité par leur adhésion commune à la CGT. A une époque où la psychopathologie du travail est encore réservée à un petit cercle d'initiés ${ }^{7}$, les membres des directions apparaissent pour leur part bien peu familiers de ce corpus.

La psychopathologie du travail de C.Dejours, qui prend le nom de psychodynamique $d u$ travail à partir de 1993, est une démarche qui s'insère avec efficacité dans les stratégies argumentatives des membres syndiqués du CNHSCT. Elle est centrée sur l'étude in situ du décalage entre les marges d'action laissées aux salariés et la responsabilité qui leur est dévolue, qui est cause de souffrance au travail. Parmi les principales causes de cette souffrance, la psychopathologie du travail pointe du doigt le fait d'évaluer le travail réel à l'aune du travail prescrit, plutôt que de constater l'écart irréductible entre les deux; également, le fait de demander à un salarié de fournir un travail sans lui en donner les moyens : injonctions contradictoires, objectifs démesurés ou encore destruction des collectifs de travail indispensables au « travailler » (Molinier, 2007 ; Dejours, 2008).

Or depuis 1985, les représentants du personnel dénoncent régulièrement la «culpabilisation», par les directions, des salariés victimes d'accidents du travail, qui

\footnotetext{
${ }^{5}$ On peut citer notamment les enquêtes distribution Lorraine (Molinier, Flottes - juin 1997), stockage gaz Germigny (Aucante, Dejours, Morice, juillet 2001), travail posté Gravelines (Molinier, Aucante, Klein, Valette décembre 2001), système électrique Rhône-Alpes Auvergne (Flottes, Robert - janvier 2002).

${ }^{6}$ Par exemple, le Docteur Christian Jayet, médecin du travail à la centrale nucléaire du Bugey, rédige en novembre 1986 un rapport intitulé "Vécu du travail au service travaux de la sous-unité technique », dans laquelle il fait part de son inquiétude quant aux problèmes psychologiques des agents. Suite à son rapport, une expertise est demandée en octobre 1987 à l'Association pour l'ouverture du champ d'investigation psychopathologique. Le rapport final, signé C. Dejours, D. Cru et D. Duclos, est rendu en juin 1988. Un schéma identique (pré-enquête du médecin, suivi d'une enquête de l'équipe de C.Dejours) se reproduit dans la plupart des demandes locales d'expertise qui vont suivre.

${ }^{7}$ Il faut attendre la parution en 1998 de l'ouvrage Souffrance en France de C.Dejours (Dejours, 1998) pour que les idées de la psychodynamique du travail soient véritablement diffusées au-delà du cercle restreint des professionnels et militants des questions de santé liées au travail.
} 
n'auraient pas respecté les préconisations élémentaires. Cette attitude a souvent pour effet de clore la discussion sur les accidents en question, sans qu'une modification des conditions de travail n'apparaisse nécessaire. Ainsi, suite à une explosion de gaz le 30 novembre 1987 rue Saint-Florentin, à Paris, qui a causé la mort d'un agent, le secrétaire M. de Coninck s'insurge contre la direction du centre de Paris-Ouest, qui aurait porté « des accusations injustifiées visà-vis des agents concernés » et entendrait clore au plus vite l'enquête. Dans ce débat récurrent sur la responsabilité des accidents du travail, la référence à la psychopathologie du travail permet aux syndicats d'invoquer une autorité scientifique pour mettre en accusation l'organisation du travail, et non le salarié pris individuellement.

Sans prétendre être un spécialiste de la psychopathologie du travail, le secrétaire $\mathrm{M}$. De Coninck reprend fréquemment ses structures argumentatives dans son discours. Lorsque C.Dejours vient exposer sa démarche au CNHSCT en février 1991, la première question que lui pose le secrétaire est: «est-il possible d'établir une relation entre les difficultés rencontrées par les agents dans l'entreprise et une démotivation qui pourrait conduire au nonrespect des règles (pouvant induire des accidents)? ", ce à quoi l'invité répond positivement. En décembre 1994, lors d'un point sur l'actualité sociale, M. De Coninck évoque en un raccourci saisissant « une justice ayant à sanctionner des routiers dont les conditions de travail sont responsables de dizaines de morts $^{8} »$. Cet usage non expert de la psychopathologie du travail permet aux représentants du personnel, lors de chaque accident grave, de mettre en accusation l'organisation du travail et de demander à participer à son amélioration.

\section{La réfutation des enquêtes psychopathologiques par les directions}

La référence à la psychopathologie du travail modifie en partie le rapport de forces entre représentants du personnel et des directions au CNHSCT. Elle permet tout d'abord de consolider les prérogatives syndicales au sein de l'instance, dont en premier lieu le droit à l'expertise. Au moment de l'accident de gaz de la rue Saint-Florentin, les directions se montraient encore réticentes à ce que les syndicats demandent une expertise, faisant remarquer que « le CNHSCT a des responsabilités de coordination mais n'est pas compétent pour faire appel à un expert» (juin 1988). Le long débat sur l'étude dans la Distribution entérine le droit syndical à demander une expertise extérieure.

La référence à la psychopathologie du travail permet également de légitimer une remise en question de l'organisation du travail dans son ensemble, qui n'en reste pas à la simple étude de l'arbre des causes qui ont mené à un dysfonctionnement précis. Pour les syndicats, l'expertise dans la Distribution est un levier pour contester le bien-fondé des « réformes de structure» lancées dans cette branche au cours des années 1980, qui réorganisent les centres de distribution en des pôles appelés « centres de résultats » (Ménard, 1997 ; Poupeau, 2001). Dans un premier temps, les directions contestent aux syndicats le droit de se prononcer sur l'organisation du travail, comme l'explique le président du CNHSCT en février 1989: «si la direction de la Distribution se propose de modifier les textes, sur des points qui ne mettent en cause que l'organisation, sans toucher aux questions de sécurité et de conditions de travail, il n'estime pas nécessaire que le CNHSCT ait à se prononcer à leur sujet ». Le secrétaire CGT répond en argumentant que " les schémas d'organisation sont bien du ressort du CNHSCT dans la mesure où ils ont des répercussions sur la sécurité et les conditions de travail ». L'histoire du Comité national montre que c'est cette acception large des prérogatives syndicales qui prévaudra jusqu'au bout, sans que les directions ne parviennent à la remettre réellement en question.

Cependant, la référence aux expertises de psychopathologie du travail ne sera pas suffisante pour que les réformes de structure soient retirées. Dès l'étude sur la Distribution, les représentants des directions contestent en effet le caractère scientifique de la démarche,

\footnotetext{
${ }^{8}$ Les routiers en question étaient à l'origine d'un carambolage meurtrier.
} 
invoquant l'absence de représentativité des expertises, menées sur quelques sites sélectionnés, quand ce n'est pas un seul site, et n'englobant que les salariés volontaires. Lors de la séance de mars 1993, le directeur de la Distribution avance ainsi que «telle qu'elle a été réalisée, cette enquête lui semble plus une généralisation d'opinions personnelles émises lors des réunions des groupes. (...) L'étude faite par le Professeur Dejours à l'agence Sambre-Avesnois n'a été réalisée qu'auprès d'un très petit nombre d'agents ».

Face à cette attaque, les représentants du personnel qui siègent au Comité national ne réussissent pas à défendre l'épistémologie de l'enquête de terrain, ou en d'autres termes, à « assumer le caractère «non représentatif» de l'entretien » (Beaud, 1996) ${ }^{9}$. Le secrétaire M. de Coninck tente plutôt d'étendre la démarche à d'autres unités, reconnaissant implicitement le bien-fondé du critère de représentativité : « il souligne ce point, qui lui paraît indispensable, pour donner une valeur plus générale à cette enquête afin qu'elle n'apparaisse pas comme un épiphénomène contestable lors de débats ultérieurs » (mars 1993). Cependant, les directions refusent de poursuivre les études, argumentant que de nouvelles enquêtes n'apporteraient rien de nouveau. Dans ce débat, la position de C. Dejours n'est d'aucun secours pour les syndicats. Raisonnant d'abord en scientifique désireux de faire progresser la connaissance, qui évolue en outre dans un champ disciplinaire où la pertinence des enquêtes de terrain n'est plus à démontrer, il se range à la position des directions : «M. Le Pr. Dejours n'est pas sûr que l'établissement d'un diagnostic d'ensemble soit intéressant. Il pense qu'il est plus important d'avoir une étude approfondie, à un endroit précis, permettant de nourrir le débat sur la transformation de l'organisation » (octobre 1993). Les études supplémentaires demandées par le secrétaire n'auront de fait jamais lieu.

\section{8-2003 : Le tournant des suicides, ou quand la souffrance devient un risque grave}

C'est à partir du début de l'année 1998 que la thématique du suicide au travail apparaît au sein d'EDF-GDF. Est-ce dû à une augmentation du nombre de suicides d'agents EDFGDF au fil du temps? A une plus grande part de suicides liés au travail ? Nous ne pouvons conclure sur ce point, qui mériterait de longs développements, dans le cadre de cette étude ${ }^{10}$. Cependant, il est certain que l'inscription de ce thème à l'ordre du jour des entreprises d'énergie se fait sur l'arrière-plan d'une médiatisation récente du lien entre suicide et travail : les cas des suicides de personnels soignants en 1995, puis celui des suicides de policiers et gendarmes en 1996-1997 font l'objet de nombreux articles de presse ${ }^{11}$.

En 1998, le débat sur le lien entre conditions de travail et suicide apparaît dans le cadre des CHSCT locaux. Il «remonte» ensuite au CNHSCT par le biais des syndicats, les membres du Comité national alertant la direction sur les informations qu'ils reçoivent de la

\footnotetext{
${ }^{9}$ L'article de Stéphane Beaud illustre le fait qu'en 1996, la légitimité d'une épistémologie proprement ethnographique, qui ne se confondrait pas avec ce que Jean-Claude Passeron appelle le « quantitatif honteux » des campagnes d'entretiens, fait encore débat même en sociologie (Beaud, 1996).

${ }^{10}$ Dans les archives du CNHSCT, le seul document faisant état de l'évolution du nombre de suicides de salariés d'EDF-GDF, durant la période 1983-2003, a été rédigé par le médecin coordonnateur en 2003 (nommé par la Direction, contrairement aux médecins délégués, élus par leurs pairs). L'enquête indique que le nombre de suicides est resté constant (autour de 30 par an) sur la période. Une déclaration d'un représentant de la CGT infirme ces données : «En tendance, il y a accroissement du fait suicidaire puisque dans la décennie 1980, le taux était de 19,78\%, et dans la décennie 1990 de 20,67\%, et que la moyenne des taux pour les années 2000 à 2002 atteint 21,63\% pour 100000 agents » (décembre 2003).

${ }^{11}$ Voir par exemple les articles parus dans le journal Le Monde : Jean-Yves Nau, « Une surveillante de l'hôpital Cochin se suicide sur son lieu de travail », Le Monde, 2 mars 1995 ; Philippe Broussard, « Le suicide est devenu la troisième cause de mortalité chez les policiers », Le Monde, 14 février 1996; Véronique Maurus, «La déprime des flics », Le Monde, 4 avril 1996 ; Jacques Isnard, « Trente et un gendarmes se sont donné la mort en 1996 », Le Monde, 27 mars 1997.
} 
part des différentes unités. Le débat sur les suicides modifie profondément les données du débat préexistant sur la souffrance psychique et la responsabilité des agents, donnant une force nouvelle à la position syndicale.

\section{Deux positions inconciliables}

Au niveau local, le débat sur les suicides d'agents EDF-GDF donne lieu à des querelles souvent violentes entre les syndicats et la Direction. Armés de la psychodynamique du travail, les représentants du personnel mettent systématiquement en cause l'organisation du travail pour expliquer l'acte de leur collègue. La Direction tente quant à elle de circonscrire le débat à un problème de psychologie individuelle, en s'aidant parfois de l'intervention d'arbitres scientifiques extérieurs; les médecins du travail d'EDF-GDF se refusant généralement à jouer le rôle d'arbitre, préférant évoquer la complexité de ces situations.

Le 19 janvier 1998, une réunion extraordinaire de CHSCT se tient à la demande des syndicats dans une unité de la Vallée de la Maurienne, suite au suicide d'un agent. Alors que les représentants du personnel mettent en cause le travail dans son passage à l'acte, le président du CHSCT (directeur de l'unité) incrimine la psychologie individuelle du salarié : « Il pense que les personnes qui se suicident ont un certain déséquilibre. Il demande qu'on lui prouve que ces personnes sont tout à fait normales ». A la question de savoir si l'agent s'est suicidé à cause des pressions de la hiérarchie ou de sa personnalité, le président répond : « Il précise que ces personnes, pouvant développer des phénomènes obsessionnels, prennent des petites peccadilles, qui au bout du compte peuvent faire tout un monde. Il admet que M. V. ait pu raconter à telle ou telle personne, qu'en rentrant de vacances, il a trouvé son bureau coupé en deux, mais pense qu'il était déjà dans un état psychologique anormal ».

Trois mois plus tard, la Direction de l'unité Vallée de la Maurienne invite un psychologue de l'université de Grenoble à une réunion de CHSCT. Il y fait un exposé sur les processus qui sous-tendent les passages à l'acte suicidaire, et soutient que les crises suicidaires sont avant tout le produit d'un certain type de personnalités génétiquement déterminées, qu'il nomme " personnalités dépressives » : « aujourd'hui, à l'âge adulte, nous ne pouvons pas dire que des conditions de travail difficiles puissent créer une personnalité dépressive ».

Le 29 janvier 1998, c'est en Haute-Vienne qu'a lieu un autre CHSCT extraordinaire,

suite là encore au suicide d'un agent. La réunion se tient dans un climat très conflictuel. Les syndicats accusent le travail d'être à l'origine de son acte : "Ses amis et représentants du personnel que nous sommes sont certains que le déclencheur est dans l'entreprise et que le mal-vivre professionnel, qui a accompagné ses dernières années de travail, est en partie responsable de sa disparition brutale ». Ils votent le voeu, qui ne sera pas suivi d'effet, que C. Dejours vienne mener une expertise psychodynamique dans leur centre. Face aux représentants du personnel, la Direction est sur la défensive, pointant du doigt les facteurs extérieurs qui peuvent expliquer cet acte : « il y a le stress du monde du travail, et il y a celui généré par la société dans laquelle nous vivons ». En fin de réunion, chaque partie se tourne vers le médecin du travail, qui apparait comme l'ultime arbitre scientifique; mais ce dernier, ne souhaitant sans doute pas trancher en faveur des syndicats ou de la Direction, se montre évasif: «On n'est pas capable de mesurer la souffrance des gens, et face à la souffrance personne ne réagit pareil ».

\section{Le travail est-il la seule cause du suicide d'un salarié?}

La stratégie principale des directions, au niveau local comme au sein du CNHSCT, est de mettre en doute les explications qui voient dans le travail la seule cause du suicide d'un salarié. Les CHSCT extraordinaires commencent ainsi généralement par une phrase du Président, qui explique avoir " accepté » que la réunion se tienne bien qu'il n'en voie pas nécessairement la pertinence, comme dans cette unité de l'Eure en mars 2002 : « bien que l'on 
n'ait pas la qualification d'accident du travail ni la reconnaissance du caractère professionnel de cet événement par la CPAM, [le Président] a accepté d'organiser cette réunion ».

Dans les débats qui s'ensuivent, les directions avancent toujours l'argument selon lequel on ne peut expliquer l'état de mal-être d'un individu par son simple travail. Lors de la séance de CNHSCT de mars 2003, le médecin coordonnateur présente à la demande des directions des « remarques générales » sur le suicide, qui insistent surtout sur le fait que c'est " un sujet particulièrement difficile » à la " nosographie complexe », dont les causes sont toujours multiples. Les représentants du personnel se montrent sensibles aux arguments sur la difficulté de démêler les causes d'un suicide. Cependant, ces réserves n'affaiblissent pas leur position, à partir du moment où ils choisissent de présenter le travail comme le facteur de déclenchement de l'acte, ou la cause aggravante d'un mal-être peut-être préexistant. En janvier 2002, dans une unité de Franche-Comté, la CGT et la CFDT font une déclaration commune qui reprend cet argument, d'une manière imagée : "nous refusons et nous ne voulons pas apprendre chaque semaine qu'un nouveau collègue a mis fin désespérément à ces jours pour fuir une vie infernale dont sans doute l'un des ruisseaux qui alimente cette rivière infernale prend sa source au sein même de nos entreprises ».

\section{Le suicide, ou la représentativité de l'individu}

Progressivement, les données de ce débat se modifient ainsi en faveur des syndicats, dans le même temps où la question des suicides liés au travail fait irruption au sein du CNHSCT. Les représentants du personnel prennent petit à petit conscience de la force nouvelle de leur argumentaire dès lors qu'il est question de suicides. Alors que les représentants des directions soulignaient le caractère non représentatif des expertises psychodynamiques, et donc du mal-être des salariés qu'elles pointaient du doigt, le suicide apparaît comme un acte suffisamment grave et violent pour ôter toute portée à cette critique. Le raisonnement implicite des syndicats est simple, et les directions ont beaucoup de difficultés à le contrer: si un salarié peut se résoudre à un tel acte, c'est qu'il souffre immensément; et si un salarié souffre à ce point, on peut supposer qu'il n'est que l'arbre qui cache la forêt de salariés confrontés à des situations de souffrance.

En décembre 2003, un cas individuel de suicide de salarié est ainsi longuement évoqué lors de la réunion du CNHSCT. La nouveauté de cette mise à l'ordre du jour doit être soulignée, les cas individuels n'étant que rarement évoqués au niveau national jusque-là, sauf dans le cas de certains accidents du travail mortels. Un représentant de la CGT lit une déclaration au sujet du suicide de Monsieur T., survenu au Centre Gard-Cévennes, dans laquelle il insiste sur la violence de l'acte :

«Dimanche 16 novembre 2003 au matin, notre collègue Monsieur T. à quitté son domicile. Son corps a été retrouvé mardi 18 novembre : il s'est donné la mort ! Un suicide est un acte extrême, d'une violence insupportable pour celui qui le commet, mais aussi pour tout son entourage (familial et professionnel).

Il poursuit avec une argumentation clairement inspirée de la psychodynamique du travail qui met en cause l'inflation règlementaire et son écart avec le travail réel, la fausse autonomie des agents qui n'ont pas les moyens de leur autonomie, et la culpabilisation des salariés :

En l'espèce, nous ne pouvons pas ignorer le lien entre la visite hiérarchique de chantier faite le vendredi 14 novembre 2003 et le suicide de notre collègue. Monsieur T. a subi un stress suffisamment fort pour qu'il puisse s'apparenter à un traumatisme psychologique. Des lettres (à son épouse et à son supérieur hiérarchique) attestent du lien de cause à effet entre les deux événements. (...) L'employeur est responsable de la sécurité de ses agents et doit aussi veiller à ce que les organisations et les conditions de travail ne portent pas atteinte à leur intégrité physique et mentale. Or, depuis quelque temps déjà, nos entreprises mettent de la règle et de la norme où il ne faudrait mettre que de la relation et du lien social. Les organisations de travail mettent les agents sous pression : (...) les agents sont sommés d'être autonomes, d'être responsables (...) et à la moindre erreur, ils sont coupables. 
Intervient alors la sanction. Nous ne pouvons tolérer que la déclinaison sur notre Centre de la note GP44A concernant le dispositif de reconnaissance/sanction soit axée essentiellement sur la répression. (...) Il est dit dans cette note qu'occulter délibérément les engagements et règles de sécurité est une faute professionnelle et que sanctionner les manquements aux règles de sécurité est un acte managérial réaffirmé. Trois manquements sont cités comme : le non-port des EPI [équipements de protection individuelle], de la ceinture de sécurité en voiture et le non-respect des procédures d'accès aux réseaux.

Messieurs les managers, vous êtes-vous interrogés pourquoi certains agents, parfois, sont amenés à ne pas respecter les consignes? Nous voulons dénoncer fermement aujourd'hui l'organisation du travail contraignante et pathogène dont vous êtes responsables.

Nous dénonçons l'exigence de résultats sans la mise à disposition des moyens nécessaires.

Nous dénonçons la surcharge de travail qui empêche les agents de penser leur travail.

Nous dénonçons la culpabilisation collective et individuelle.

Nous dénonçons la démarche tendant à nier le lien de causalité entre le travail et le suicide de Monsieur T. »

Cette charge violente contre les directions ne suscite aucune contestation de leur part, le Président du CNHSCT se contentant de rappeler l'engagement du management sur la question de la souffrance psychique des salariés. Cette réponse est emblématique du retournement qui a eu lieu dans le débat sur la souffrance : représentants du personnel et des directions s'accordent désormais à considérer la souffrance psychique comme un risque lourd ou grave, dont la prévention vaut pour elle-même.

La déclaration de la CGT et la réaction des directions témoignent également du déplacement du débat sur la responsabilité. L'agent qui se suicide, tout comme l'agent victime d'un accident du travail grave, sont présentés comme les victimes d'un management pathogène. Dans les débats du CNHSCT, il n'apparait plus possible de mettre hors de cause la responsabilité des directions dans la souffrance psychique et les accidents du travail des salariés. L'évolution de la jurisprudence ira d'ailleurs dans ce sens, de plus en plus de suicides de salariés étant reconnus comme des accidents du travail ou des maladies professionnelles, et la « faute inexcusable » des directions étant de plus en plus souvent mise en cause devant les tribunaux, notamment chez EDF-GDF ${ }^{12}$.

\footnotetext{
${ }^{12}$ Le Dr. Dominique Huez, médecin du travail de la centrale nucléaire de Chinon, a notamment contribué à cette évolution de la jurisprudence (Huez, 2008). Voir également Xavier Renard, « EDF : un suicide reconnu comme maladie professionnelle », Libération, 17 avril 2009.
} 


\section{3-2008 : Le débat sur les risques psychosociaux}

La mobilisation syndicale autour des suicides de salariés continue tout au long des années 2000, notamment autour de la centrale nucléaire de Chinon. Cependant, à partir de 2003, les directions d'EDF et de GDF inaugurent une reprise en main du thème de la souffrance psychique des salariés au sein des instances nationales des entreprises, pour parer au renforcement juridique de la responsabilité de l'employeur. Le concept de risques psychosociaux, étroitement lié aux nouveaux groupes de travail managériaux qui la portent, autonomise le traitement de la souffrance psychique par rapport au débat classique sur la responsabilité des maux du travail.

\section{Un renforcement de la responsabilité de l'employeur}

Par la gravité des actes commis, l'émergence du problème des suicides de salariés donne davantage de force à l'argument selon lequel l'employeur est responsable de l'état psychique de ses salariés. Deux évolutions du droit renforcent cette tendance. D'une part, le décret du 5 novembre 2001 oblige l'employeur à établir un « document unique d'évaluation des risques » sous peine de sanctions pénales. Les employeurs se trouvent dans l'obligation d'établir qu'ils ont, à tout le moins, organisé la recension et la prévention des risques professionnels, quand bien même ils ne les font pas disparaître. D'autre part, en février 2002, les «arrêts amiante » de la chambre sociale de la Cour de Cassation modifient la notion de faute inexcusable de l'employeur (prévue par la loi de 1898 sur les accidents du travail) dans un sens favorable aux victimes : ceci ouvre la voie à de nombreux recours victorieux de salariés victimes d'accidents du travail et de maladies professionnelles, qui souhaitent obtenir une majoration d'indemnisation et, à défaut de pouvoir attaquer au pénal, faire reconnaître au moins symboliquement par la justice la responsabilité de l'employeur ${ }^{13}$ (Margraff et al, 2006).

Ces évolutions sont suivies de très près par le management d'EDF-GDF. Une note juridique de 2003 relève ainsi que " les arrêts ne laissent aucun doute, les tribunaux retiendront la faute caractérisée de l'employeur ou de son représentant, à chaque fois qu'ils relèveront une infraction au code du travail ou un manquement qui entre dans les prérogatives du chef d'établissement, quand bien même aurait-il délégué l'application des règles de sécurité à des collaborateurs $»^{14}$.

\section{Les " risques psychosociaux », concept d'entreprise?}

C'est dans ce contexte que la direction d'EDF ${ }^{15}$ lance en 2003 une «politique de santé-sécurité » dans laquelle apparaît la notion de risques psychosociaux. En septembre 2003, un membre du Service de maîtrise des risques au travail $\left(\mathrm{SMaRT}^{16}\right)$ et un médecinconseil de la direction ${ }^{17}$ viennent présenter cette politique lors d'une séance du CNHSCT. Selon le médecin-conseil, l'un des objectifs de cette démarche est la prévention de risques considérés comme nouveaux ou émergents, dont les risques psychosociaux. Le médecinconseil définit ces derniers par une énumération d'éléments divers : " troubles anxiodépressifs, violences et agressions, harcèlement moral ou sexuel, suicides, stress ». Il ajoute

\footnotetext{
13 Selon l'avocat Michel Ledoux, « la faute inexcusable ne se résume pas à une banale procédure d'indemnisation ; aux yeux de nombre d'employeurs, c'est même un gros mot. Et pour bien des salariés, c'est un moyen d'individualiser, de montrer du doigt l'entreprise fautive, condamnée à indemniser celui qu'elle a rendu malade. Bon nombre de victimes y tiennent absolument, quitte à perdre du temps » (cité in. Le Garrec, 2006).

${ }^{14}$ Note juridique du Service de maîtrise des risques au travail. Les auteurs soulignent.

${ }^{15}$ Et non les directions d'EDF-GDF : les deux entreprises sont de plus en plus dissociées au fil du temps.

${ }^{16}$ Le SMaRT est créé en 2003, prenant la suite de la Délégation prévention gestion des risques.

${ }^{17}$ Les médecins-conseil travaillent au sein d'une structure d'expertise rattachée à la direction de la santé et de la sécurité du groupe.
} 
que « le souci était de ne pas démarrer par des idées ou des concepts qui rencontreraient une opposition dès le départ $»$.

En septembre 2005, un membre du SMaRT revient parler des risques psychosociaux lors d'une séance du CNHSCT : il explique qu'il a reçu une commande des directions d'EDF et GDF pour « établir une méthode pour caractériser et traiter la situation » et « développer un outil d'évaluation du risque ». La définition des risques psychosociaux élaborée par son service est de nouveau une liste, déclinée en sept items : "violence/agression; violence interne; harcèlement; stress au travail; troubles anxieux et dépressifs; traumatismes collectifs ; dépression »

Pour les directions, la notion de risques psychosociaux présente l'avantage de transposer la souffrance psychique sur le plan du risque, évaluable dans le cadre du document unique d'évaluation. Telle qu'elle a été présentée au CNHSCT, la notion entretien un rapport assez distant avec la littérature scientifique. Certes, la présentation des risques psychosociaux comme une liste d'éléments fait écho par son caractère éclaté à certaines définitions autorisées, comme celle proposée par l'INSERM dès 2000 : « les facteurs psychosociaux au travail désignent un vaste ensemble de variables qui se situent à l'intersection des dimensions individuelle, collective et organisationnelle de l'activité professionnelle, d'où leur complexité et leur caractère souvent composite $»^{18}$. Il faut souligner néanmoins que les documents managériaux présentés dans cette période ne cherchent pas à fonder scientifiquement la légitimité de la notion de risques psychosociaux, ni ne suivent l'évolution des débats scientifiques à son sujet - au contraire du concept de "souffrance au travail », que les représentants des salariés rapportaient sans cesse aux travaux de Dejours. Les travaux fondateurs de Robert Karasek et Joseph Siegrist (Siegrist, 1982 ; Karasek, 1990) ne sont cités que pour l'élaboration de questionnaires d'évaluation du niveau des risques. La notion de risques psychosociaux apparaît comme un concept d'entreprise, distinct du concept élaboré et discuté dans le débat scientifique ; son sens, jamais stabilisé, est à chercher non pas dans une phrase employant le présent de vérité générale, mais dans l'analyse des effets qu'il provoque, des actions qu'il rend possibles, dans l'entreprise.

La notion de concept d'entreprise renvoie également au fait que, contrairement aux deux moments précédents du débat sur la souffrance psychique, ce sont exclusivement les représentants de la direction, et les experts qui travaillent avec elle, qui suscitent ici le changement des termes de la discussion. Les risques psychosociaux sont amenés au sein du CNHSCT par les nouveaux services centraux créés dans les années 2000, tel le SMaRT, plutôt que par les représentants des salariés, qui se retrouvent dans une position de spectateurs.

\section{Les effets d'une expression}

L'utilisation de la notion de risques psychosociaux par le management produit trois effets majeurs : une autonomisation du débat sur la souffrance psychique par rapport à celui sur les risques physiques; le passage d'une obligation de prévention à une obligation d'évaluation - qui va dans le sens de l'obligation d'établir le document unique d'évaluation des risques; enfin, le remplacement du CNHSCT par des groupes de travail managériaux pour toutes les décisions concernant la souffrance psychique.

La question de la souffrance psychique était apparue, on l'a vu, comme un argument supplémentaire au sein d'un débat déjà existant sur la part de responsabilité du salarié et de l'employeur dans les accidents du travail. Or, dans un débat posé en termes de risques psychosociaux, plusieurs éléments concourent à rendre plus difficile l'imputation des responsabilités des accidents du travail, des suicides ou de la souffrance psychique. La distinction entre des victimes et des responsables de la souffrance psychique cède la place à

\footnotetext{
${ }^{18}$ Voir INSERM, 2000, $\mathrm{p}: 84$.
} 
une distinction entre des individus et un environnement risqué. D'ailleurs, pour les directions, le flou de la notion de risques psychosociaux est pensé comme un facteur de consensus permettant d'échapper à la recherche des coupables ${ }^{19}$. Enfin, dans le schème de l'évaluation des risques qui façonne la politique des directions, l'item «risques psychosociaux » est juxtaposé à l'item « risques physiques », comme s'il s'agissait de deux ordres indépendants de phénomènes.

Par ailleurs, dans la période où apparaît le vocable de risques psychosociaux, les participants aux réunions du CNHSCT s'accordent progressivement sur le fait que l'évaluation des risques est déjà une réponse à ces derniers, la prévention n'étant qu'une étape ultérieure (et plus complexe). Comme l'explique en septembre 2003 le directeur du SMaRT, les risques psychosociaux, et plus généralement la catégorie des "risques émergents ", sont définis selon son service par le fait qu'ils appellent une réponse : «Répondant à une question de M.Gerber, M.Vacher précise qu'un risque émergent est un risque qui est identifié comme devenant un problème et qui ne fait pas encore l'objet d'une action particulière, mais dont le niveau semble devoir à court terme en entraîner une ». Au fur et à mesure des réunions, l'évaluation devient en soi une réponse valable aux risques psychosociaux, non seulement du point de vue des directions, mais également des syndicats. En décembre 2003, la CGT et Force Ouvrière votent une résolution lors de la séance du CNHSCT qui se contente de demander une inscription dans le document unique d'évaluation : «nous revendiquons que l'employeur remplisse toutes ses obligations en matière de risques psychosociaux et que la prévention de ces risques soit dûment intégrée dans le document unique ». On peut remarquer, en outre, que le caractère protéiforme et flou de la notion de risques psychosociaux renforce cette tendance à limiter l'objectif de prévention, tant à chaque réunion chacun s'accorde sur la nécessité préalable de définition - ce qui occupe une bonne partie des séances.

Un troisième aspect de la modification des données du débat est la marginalisation du CNHSCT dans le traitement de la souffrance psychique. L'élaboration «maison » d'un concept de risques psychosociaux par le management s'est accompagnée d'une prolifération de groupes de travail qui regroupent essentiellement des managers et des experts; quand des représentants des salariés y sont associés, ils n'y restent généralement pas longtemps, rebutés par la démarche et le langage utilisés.

Ainsi, le «dispositif d'entreprise » de prévention des risques psychosociaux de septembre 2005 comprend un groupe du travail du SMaRT et un "groupe miroir » (comprenant dix représentants du collège managers "Ressources humaines", des médecins et des élus du CNHSCT) qui n'a qu'un avis consultatif. Lorsque les représentants des salariés demandent à avoir accès à davantage de documents sur ce dispositif de prévention, le président «précise qu'il n'est pas prévu, dans le déploiement, que les documents associés passent en CNHSCT ». Lors d'une présentation des résultats de la politique de santé-sécurité en février 2007, le secrétaire du CNHSCT Joseph Boyer se montre critique : " il a le sentiment que les directions se fabriquent un environnement virtuel dans lequel elles fonctionnent avec des indicateurs plus ou moins fiables ». Enfin, lors d'une séance en novembre 2007, les représentants de la direction annoncent le développement d'une démarche de benchmarking (évaluation) des unités sur la prévention des risques, en les comparant à d'autres entreprises équivalentes. Un représentant de la CGT déplore alors que les conclusions des audits réalisés dans le cadre de cette politique restent confidentielles : « les CHSCT, notamment, doivent en être informés (...) Il a l'impression que le déploiement

\footnotetext{
${ }^{19}$ C'est ce que reconnaissent le psychiatre Patrick Légeron et le magistrat Philippe Nasse dans leur « Rapport sur la détermination, la mesure et le suivi des risques psychosociaux au travail », remis au ministre du travail en 2008. Alors qu'auparavant, «l'accent mis sur les causes se transpos[ait] à la recherche de la responsabilité des fauteurs de ces causes et toute possibilité de consensus disparaî[ssait] à l'origine » (Nasse et Légeron, 2008, p.6), la catégorie de risques psychosociaux rendrait à l'inverse possible le « consensus d'approche » nécessaire à la prise en charge de la souffrance au travail.
} 
de la Politique Santé-Sécurité se limite au collège management sans redescendre sur le terrain $\gg$.

La marginalisation du CNHSCT sur la question de la souffrance psychique s'inscrit dans un mouvement plus général de reprise en main des questions de santé au travail par le management. En 2007 est créé l'Observatoire de la qualité de vie au travail, piloté par la direction d'EDF ; il est présenté comme le premier pas d'un projet d'entreprise qui donne un cadre nouveau aux questions de santé, sécurité et conditions de travail, associant là médecins, managers, experts et représentants du personnel, dans une arène peu formalisée et qui n'a pas le statut d'une instance représentative du personnel. Quelques mois plus tard, les directions annoncent la suppression du CNHSCT, arguant de la réforme d'ensemble des instances représentatives du personnel ${ }^{20}$. La séance du 31 janvier 2008 donne le coup d'arrêt à la dernière instance représentative du personnel d'échelle nationale.

\section{Conclusion}

L'analyse des débats du CNHSCT a permis de dégager trois temps du traitement de la souffrance psychique des salariés au sein des entreprises EDF et GDF.

Dans un premier moment (1985-1998), la problématique de la souffrance psychique est mobilisée par les représentants des salariés dans le cadre du débat préexistant sur la responsabilité des accidents du travail. La psychodynamique du travail permet de montrer l'irresponsabilité du salarié, à qui l'on ne donne pas les moyens de faire son travail correctement, et de faire porter la faute des accidents sur l'organisation du travail, et donc sur l'employeur qui décide de cette dernière.

Le moment de la première crise des suicides (1998-2003), par la gravité des actes commis, fait pencher encore davantage la balance de la responsabilité du côté de l'employeur : si un salarié est prêt à se suicider, c'est qu'un grand nombre de salariés sont en dépression du fait de leur travail, argumentent les représentants du personnel. À l'instar des électrocutions et des explosions de gaz, la souffrance psychique devient également en ellemême un risque lourd, entraînant la mort, et implique dès lors inévitablement une recherche de responsabilité spécifique.

Le troisième moment (2003-2008) est celui de la reprise en main du thème de la souffrance psychique par les instances de direction. Le concept de " risques psychosociaux », inséparable de nouvelles institutions qui le portent parallèlement à l'activité du CNHSCT, autonomise le débat sur la souffrance psychique par rapport au débat sur la responsabilité des risques : l'activité principale des instances de prévention devient alors de définir le concept de risques psychosociaux et de le quantifier dans des études menées par des groupes de travail à majorité managériale, qui évincent progressivement le CNHSCT de ce domaine, jusqu'à sa suppression en janvier 2008.

Dans les instances de type CHSCT, la direction et les syndicats ne sont pas mûs par de simples considérations sanitaires ou hygiénistes. La mobilisation dans l'enceinte de l'entreprise de concepts empruntés à la recherche scientifique est fonction du soutien qu'ils apportent à des débats déjà en cours sur d'autres enjeux : ici, le débat sur le partage de la responsabilité des accidents. Ce soutien dépend à la fois de la structure argumentative des théories et concepts mobilisés - qui permet ou non, ici, certaines imputations de responsabilité - mais également des ressources à la disposition de ceux qui portent ces idées.

\footnotetext{
${ }^{20}$ Dès 2004, les entreprises EDF S.A. et Gaz de France sont en effet entrées dans le droit commun, et commencent à séparer certains de leurs services et de leurs fonctions restés jusque-là « mixtes » (appartenant à la fois à EDF et GDF). C'est le cas des services de santé et de prévention, mais aussi de la distribution, transformée en deux filiales, indépendantes juridiquement des entreprises-mères.
} 


\section{Bibliographie}

Beaud S. 1996. « L'usage de l'entretien en sciences sociales. Plaidoyer pour l'entretien ethnographique », Politix, n³5, p. 226-257.

Dejours C. 1998. Souffrance en France. La banalisation de l'injustice sociale, Paris : Seuil.

Dejours C. 2008 [1980]. Travail, usure mentale : essai de psychopathologie du travail. Paris : Bayard.

Dodier N. 1999. «La construction sociale des souffrances corporelles dans les relations quotidiennes de travail ». In Dejours C. (dir.). Plaisir et souffrance dans le travail. Paris : Editions de l'AOCIP.

Freidson E. 1984. La profession médicale. Paris : Payot.

Gollac M. et al. 2006. « Du déni à la banalisation. Note de recherche : sur la souffrance mentale au travail ». Actes de la recherche en sciences sociales, $\mathrm{n}^{\circ} 163$, p.39-45.

Hatzfeld N. 2006a. "Ergonomie, productivité et usure au travail. Une décennie de débats d'atelier à Peugeot-Sochaux (1995-2005), Actes de la recherche en sciences sociales, $\mathrm{n}^{\circ} 165$, p.93-105.

Hatzfeld N. 2006b. « Entre fonction technique et démocratie participative, Les débuts discutés des CHS (1947-1970) », Les politiques du travail (1906-2006), acteurs, institutions réseaux, (sous la direction de Alain Chatriot, Odile Join-Lambert et Vincent Viet). Rennes : PUR, p.403-415.

Henry E. 2000. Un scandale improbable. Amiante : d'une maladie professionnelle à une "crise de santé publique ». Thèse, UTC Compiègne.

Henry E. 2003. « Intéresser les tribunaux à sa cause : contournement de la difficile judiciarisation du problème de l'amiante ", Sociétés contemporaines, n ${ }^{52}$, p.39-59.

Holmes S. 2006. « «Parce qu'ils sont plus près du sol». L'invisibilisation de la souffrance sociale des cueilleurs de baies ». Actes de la recherche en sciences sociales, n¹65, p.28 à 51 .

Huez D. 2008. Souffrir au travail. Comprendre pour agir. Paris : Privé.

Institut National de Recherche et de Sécurité pour la prévention des accidents du travail et des maladies professionnelles. 2005. "L'évaluation des risques professionnels ». Paris : INRS.

Institut National de la Santé et de la Recherche Médicale. 2000. « Lombalgies en milieu professionnel.Quels facteurs de risque et quelle prévention? » Rapport d'expertise collectif, éditions de l'INSERM.

Karasek R., Theorell T., 1990. Healthy Work. Stress, Productivity and the Reconstruction of Working Life. New York : Basic Books.

Le Garrec J. et al. 2006. « Rapport fait au nom de la mission d'information sur les risques et les conséquences de l'exposition à l'amiante », Paris : Assemblée nationale.

Margraff A., Graser M, Manaouil C. 2006. "Prise en charge du suicide au titre de la réglementation sur les accidents du travail ». Archives des maladies professionnelles et de l'environnement, $\mathrm{n}^{\circ} 67, \mathrm{p} .513-520$.

Ménard, J.-Y., Barreau, J., 1997. Stratégies de modernisation et réactions du personnel. Le cas de trois entreprises publiques d'électricité : Électricité De France, Hydro-Québec \& Société Hongroise d'Électricité. Paris, L'Harmattan. 
Molinier P. 2007. Les enjeux psychiques du travail : introduction à la psychodynamique du travail. Paris : Payot.

Nasse P. et Légeron P. 2008, Rapport sur la détermination, la mesure et le suivi des risques psychosociaux au travail. Paris, La documentation française.

Omnès C. et Pitti, L. (dir.) 2009, Cultures du risque au travail et prévention au XXe siècle. La France au regard des pays voisins. Rennes, Presses Universitaires de Rennes.

Poupeau F.-M. 2001. "Libéralisation du service public et action publique locale. Le département dans la recomposition du système de distribution électrique français ». Sociologie du Travail, Vol. 43-2, p. 179-195.

Rosental P.-A. 2007. « Avant l'amiante, la silicose. Mourir de maladie professionnelle dans la France du XXe siècle ». Population et sociétés, $\mathrm{n}^{\circ} 437$.

Rosner D. et Markowitz G. 2009. «L'histoire au prétoire. Deux historiens dans les procès des maladies professionnelles et environnementales ", Revue d'histoire moderne et contemporaine, $\mathrm{n}^{\circ} 56-1$, p. 227-252.

Siegrist J., Dittmann K. Weidemann H. 1982. « The role of psychosocial risks in patients with early myocardial infarction », Activitas Nervosa Superior (Praha), 24(1), p.14-24. 